\title{
Working memory and inattentional blindness
}

\author{
Keith Bredemeier • Daniel J. Simons
}

Published online: 6 January 2012

(C) Psychonomic Society, Inc. 2011

\begin{abstract}
Individual differences in working memory predict many aspects of cognitive performance, especially for tasks that demand focused attention. One negative consequence of focused attention is inattentional blindness, the failure to notice unexpected objects when attention is engaged elsewhere. Yet, the relationship between individual differences in working memory and inattentional blindness is unclear; some studies have found that higher working memory capacity is associated with greater noticing, but others have found no direct association. Given the theoretical and practical significance of such individual differences, more definitive tests are needed. In two studies with large samples, we tested the relationship between multiple working memory measures and inattentional blindness. Individual differences in working memory predicted the ability to perform an attentiondemanding tracking task, but did not predict the likelihood of noticing an unexpected object present during the task. We discuss the reasons why we might not expect such individual differences in noticing and why other studies may have found them.
\end{abstract}

Keywords Inattentional blindness · Attention · Working memory $\cdot$ Capacity $\cdot$ Individual differences

Inattentional blindness is the failure to notice an unexpected object when attention is focused elsewhere (Mack \& Rock, 1998). Many studies have explored how the stimuli and the task parameters affect noticing, but few have explored individual differences in noticing, in part because of the inability to test the

\footnotetext{
K. Bredemeier $(\square) \cdot$ D. J. Simons

Department of Psychology,

University of Illinois at Urbana-Champaign,

Urbana, IL, USA

e-mail: kbredem2@illinois.edu

D. J. Simons

e-mail: dsimons@illinois.edu
}

same individual with the same unexpected object repeatedly; once participants are questioned about an unexpected object, it will be expected on subsequent trials. Without the possibility of multiple trials with the same individual, the only means to study individual differences is to test large numbers of participants.

Given the demands of typical inattentional-blindness tasks, if individual differences do predict noticing, one of the best candidate predictors would be working memory capacity. Working memory predicts performance on a wide range of cognitive tasks, especially those that demand focused attention (see Unsworth \& Engle, 2007). Also, taxing an individual's working memory increases his or her susceptibility to inattentional blindness (Fougnie \& Marois, 2007; Todd, Fougnie, \& Marois, 2005). If people with greater working memory capacity have more resources available when performing an attentiondemanding task, they may be more likely to notice an unexpected object. Alternatively, people with better working memory capacity might be better able to maintain their focus on a task without being distracted. If so, they might be less likely to notice unexpected objects, because they can filter out irrelevant distractions (see Conway, Cowan, \& Bunting, 2001; Conway \& Kane, 2001; Lavie \& De Fockert, 2005). In fact, more recent evidence has contradicted the earlier findings, by showing that taxing working memory decreases rather than increases inattentional blindness (De Fockert \& Bremner, 2011).

Whether or not working memory load increases inattentional blindness, individual differences in working memory might be unrelated to the detection of unexpected objects. Working memory capacity predicts performance on attention-demanding tasks (Engle, 2002), including the ability to ignore known distractors. However, the processes that lead to such orienting might differ from those responsible for the detection of unexpected objects (Most \& Simons, 2001). For example, abrupt onsets influence the orienting of attention, but when they occur unexpectedly, they are not noticed more often than gradual onsets (Most, Scholl, Clifford, \& Simons, 2005). Furthermore, working memory 
does not play a critical role in all controlled attention processes (Kane, Poole, Tuholski, \& Engle, 2006).

Only a handful of published studies have examined the relation between working memory and inattentional blindness (Hannon \& Richards, 2010; Richards, Hannon, \& Derakshan, 2010; Seegmiller, Watson, \& Strayer, 2011), and they have produced conflicting results. Consistent with the idea that additional capacity enhances noticing, people with greater working memory capacity were more likely to notice an unexpected red cross in a sustained inattentional-blindness task (Hannon \& Richards, 2010; Richards et al., 2010). Those two studies used fairly heterogeneous samples, though, allowing for the possibility that the relationship they described was affected by other factors (e.g., age). Another recent study (Seegmiller et al., 2011) found no overall relationship between working memory and noticing of an unexpected gorilla (Simons \& Chabris, 1999), though it did find a relationship for a selected subset of participants who performed the primary attention task with high accuracy.

Given the theoretical and practical importance (see, e.g., Chabris, Weinberger, Fontaine, \& Simons, 2011; Strayer \& Drews, 2007) of individual differences in the detection of unexpected objects, it is essential to test the scope and reliability of such differences. In light of the somewhat mixed evidence for a relationship between working memory capacity and noticing in the initial studies, we conducted two large-scale individual-differences studies using a sustained inattentionalblindness task and multiple measures of working memory.

\section{Study 1}

\section{Method}

Participants College students $(N=134)$ with normal or corrected-to-normal vision participated in exchange for course credit $(58 \%$ female; mean age $=19.0$ years, $S D=1.0$, range $=$ 18-22). The tasks reported here were part of a larger study of the relationship between emotional distress and attention for which the participants completed other, unrelated tasks and measures (selective attention tasks, as well as personality and distress questionnaires). Participants were tested individually.

Materials and procedure Each participant first completed verbal and spatial $n$-back tasks (in counterbalanced orders) as a measure of working memory (Owen, McMillan, Laird, $\&$ Bullmore, 2005). For each task, 20 capital and lowercase letters appeared sequentially for $500 \mathrm{~ms}$ each (2-s delay between letters) at one of 10 possible locations on a computer monitor; participants pressed " $\mathrm{s}$ " if the current item matched the one presented two items earlier, or "d" if it did not. For the verbal $n$-back task, participants judged whether the letter was the same as the letter two back. For the spatial $n$-back task, they judged whether the letter was in the same location. Nonresponses were treated as errors. Participants completed five trials of each type, and the first trial for each was treated as practice, resulting in a working memory measure based on 72 responses for each task.

Following the $n$-back tasks, participants completed a sustained-attention task in which they counted the total number of times that four moving white shapes (two Ls, two Ts) touched the sides of a $640 \times 480$ window centered on a 15 -in. monitor (resolution, $1,024 \times 768$; viewing distance approximately $60 \mathrm{~cm}$ ) while ignoring the movements of four black Ls and Ts. Each trial lasted $12 \mathrm{~s}$ and was followed by a prompt to type the total number of touches by the white shapes. Given that objects sometimes entered a corner and bounced twice in rapid succession, attentive observers still might miss an occasional touch. Consequently, the total counts were treated as accurate if they were within $20 \%$ of the actual number of bounces (rounding up; see Simons \& Jensen, 2009, for more detail).

After four trials with no unexpected events, the participants completed a critical trial: After $4 \mathrm{~s}$ of object motion, a gray cross entered the display vertically centered on the right edge, moved horizontally across the display, and exited on the left after $4 \mathrm{~s}$. We used a gray cross as the unexpected object with the expectation that about $50 \%$ of participants would notice it (Most et al., 2001). All objects moved at a speed of 176 pixels/ $\mathrm{s}$, and the motion of the letters continued for $4 \mathrm{~s}$ after the cross disappeared. After reporting the number of touches, participants were asked "On this trial, did you see anything other than the black and white Ls and Ts (anything that was not present on the first 4 trials)?" If they responded "yes," they were asked to type what they saw into a text field. Those who accurately described the shape, color, or motion of the unexpected stimulus were considered to have noticed it, whereas those who reported nothing unexpected were considered to have experienced inattentional blindness.

\section{Results and discussion}

For the detailed results, see Table 1. Overall, 89 participants (70.6\%) noticed the unexpected stimuli. ${ }^{1}$ Counting accuracy across the four noncritical trials in the sustained-attention task was used as an index of tracking ability. ${ }^{2}$ The data from participants who showed near-chance performance (i.e., below $60 \%$ ) on the $n$-back tasks were excluded from the

\footnotetext{
${ }^{1}$ Eight of the participants reported noticing something unexpected on that trial but did not accurately describe the unexpected stimulus, and thus were excluded from the analyses. The results were virtually identical when these individuals were treated as noticers.

${ }^{2}$ Our results did not change when other approaches for computing tracking accuracy were used (e.g., absolute accuracy, relative accuracy).
} 
Table 1 Means (and standard errors in parentheses) for participants who did and did not notice the gray cross, along with test statistics and $p$ values from the logistic regression analyses

\begin{tabular}{lrllll}
\hline & $n$ & Noticed Cross & Missed Cross & Wald Statistic & $p$ Value \\
\hline Study 1 & & & & & \\
Tracking accuracy (\% correct) & 126 & $59.3(3.4)$ & $62.2(4.6)$ & 0.23 & .63 \\
$N$-back, letter version (\% correct) & 111 & $84.8(1.1)$ & $86.4(1.4)$ & 0.65 & .42 \\
$N$-back, letter for accurate counters & 50 & $84.4(1.8)$ & $87.3(2.0)$ & 0.94 & .33 \\
$N$-back, spatial version (\% correct) & 107 & $82.9(1.1)$ & $84.7(2.0)$ & 0.67 & .41 \\
$N$-back, spatial for accurate counters & 51 & $84.5(1.5)$ & $85.9(2.3)$ & 0.28 & .60 \\
Study 2 & & & & & \\
Tracking speed (in pixels/s) & 195 & $137.4(2.9)$ & $136.6(1.9)$ & 0.05 & .83 \\
$N$-back, letter version (\% correct) & 174 & $87.9(0.9)$ & $88.1(0.6)$ & 0.01 & .94 \\
$N$-back, letter $\times$ tracking speed & 174 & $0.25(0.11)$ & $0.09(0.08)$ & 1.36 & .24 \\
$N$-back, spatial version (\% correct) & 170 & $87.0(1.1)$ & $85.8(0.7)$ & 0.75 & .39 \\
$N$-back, spatial $\times$ tracking speed & 170 & $0.20(0.13)$ & $0.22(0.10)$ & 0.00 & .97 \\
Automated OSPAN scores & 195 & $39.5(2.5)$ & $43.3(1.4)$ & 1.91 & .17 \\
OSPAN scores $\times$ tracking speed & 195 & $0.14(0.14)$ & $0.12(0.08)$ & 0.00 & .96 \\
\hline
\end{tabular}

working memory analyses ( 15 for the verbal $n$-back task and 19 for the spatial $n$-back task). We conducted logistic regression to determine whether tracking accuracy or working memory performance predicted noticing of the unexpected object. As in earlier studies (Simons \& Jensen, 2009), individual differences in tracking accuracy did not significantly predict noticing $(p=.63)$. Consistent with the overall analysis of Seegmiller et al. (2011), but inconsistent with other evidence (Hannon \& Richards, 2010; Richards et al., 2010), individual differences in working memory performance also did not predict noticing (all $p s>.4$ ). ${ }^{3}$ Inconsistent with Seegmiller et al.'s results, however, working memory still did not predict noticing when we included only participants who counted touches accurately on the critical trial (all $p \mathrm{~s}>.3$ ).

Both tracking and $n$-back tasks rely on executive control, and performance on the $n$-back tasks was positively correlated with tracking (verbal $n$-back, $r=.27, p<.01$; spatial $n$ back, $r=.21, p<.05)$. However, none of these tasks was related to the detection of the unexpected object, suggesting that the capture of awareness by unexpected objects might not rely on the same mechanisms involved in other focused attention tasks.

\section{Study 2}

One discrepancy between our approach and that of studies finding a relationship between working memory and noticing was our use of $n$-back tasks to measure working memory,

\footnotetext{
${ }^{3}$ We explored various approaches for calculating performance for the working memory tasks (e.g., using a different minimum accuracy cutoff). We also computed a composite working memory score by standardizing and averaging scores from the $n$-back tasks. None of these efforts yielded a significant association between working memory and noticing.
}

a paradigm that some have argued lacks construct validity (Kane, Conway, Miura, \& Colflesh, 2007). In Study 2, we attempted to replicate our finding using a larger sample and an additional measure of working memory: the operation span task (OSPAN). Another shortcoming of Study 1-one shared by earlier studies linking working memory and noticing - was the use of a relatively noisy measure of tracking ability: That is, tracking accuracy was computed from a small number of trials. In Study 2, we used an adaptive staircase technique to provide a better measure of tracking ability (Simons \& Jensen, 2009), thereby allowing a stronger test of whether tracking ability moderates the relationship between working memory and noticing. Finally, in Study 2 we checked whether our participants were familiar with inattentional blindness; many studies exclude participants with knowledge of inattentional blindness (e.g., Most et al., 2001; Richards et al., 2010), although the differences between our task and better-known variants should mitigate this concern (see Beanland \& Pammer, 2010).

\section{Method}

Participants College students $(N=207)$ with normal or corrected-to-normal vision participated in the study $(56 \%$ female; mean age $=19.5$ years, $S D=1.7$, range $=18-26$ ). Of these participants, 167 received course credit, and the remaining 40 were recruited via flyers and paid $\$ 10 / \mathrm{h}$ in exchange for their participation (the flyers targeted people with a history of anxiety or depression). As in Study 1, the tasks were part of a larger study on emotional distress and cognitive functioning, and participants completed other, unrelated tasks and measures (selective attention and executive function tasks, personality and distress questionnaires, and a diagnostic interview). Participants were tested individually. 
Materials and procedure Each participant completed the verbal and spatial $n$-back tasks, an automated version of the OSPAN task (Unsworth, Heitz, Schrock, \& Engle, 2005), and a sustained inattentional-blindness task in a randomly determined order. The $n$-back tasks were identical to those of Study 1. On each trial of the OSPAN task, participants solved a set of arithmetic operations (e.g., " $(6 \times 2)-5=$ ??”) as quickly as possible while remembering a letter that followed each equation. After each set of problems, they recalled all of the letters in order (with accuracy feedback). The number of letters in each set varied from three to seven, and participants completed three trials of each set size. The OSPAN score was the sum of all perfectly recalled sets (possible range: 0-75). Automated OSPAN scores have excellent test-retest reliability and are highly correlated with scores from the traditional OSPAN task (Unsworth et al., 2005).

Except as noted, the sustained-attention task and stimuli were identical to those of Study 1, although the procedure differed. Specifically, after five practice trials, participants completed a block of trials in which object speeds were adjusted adaptively on the basis of tracking accuracy. If the total count on a trial was within $20 \%$ of the correct total (rounding up), the objects moved faster on the next trial. If not, they moved slower. Object speed was adjusted in this manner to determine the speed (in pixels/s) at which each participant could count accurately on $75 \%$ of trials (i.e., their tracking ability). Immediately after this adaptive block, participants completed four trials on which all of the items moved at a fixed speed (150 pixels/s), followed by one critical trial: After $3 \mathrm{~s}$, a gray cross entered the display vertically centered on the right edge, moved horizontally across the display, and exited on the left after $4.7 \mathrm{~s}$. The letters continued moving for $500 \mathrm{~ms}$ after the cross disappeared. The cross was slightly darker than in Study 1, in the hopes of increasing the rate of inattentional blindness.

During debriefing, participants were asked whether they had heard of the concept of inattentional blindness (and if so, to describe it in their own words) and whether they had ever seen any inattentional-blindness demonstrations (e.g., "gorilla in our midst"; Simons \& Chabris, 1999). Clarification questions were asked as needed.

Results and discussion

See Table 1. In this sample, 53 participants (27.2\%) reported noticing the unexpected stimulus and provided an adequate description. ${ }^{4}$ The data from participants who showed near-

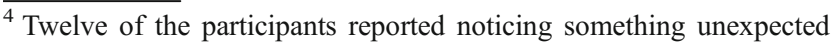
on that trial but did not accurately describe the unexpected stimulus, and thus were excluded from the analyses. Again, our results were virtually identical when these individuals were treated as noticers.
}

chance performance (i.e., below $60 \%$ ) on the $n$-back tasks were excluded from the working memory analyses ( 21 for the verbal $n$-back task and 25 for the spatial $n$-back task). Many of the participants were familiar with inattentional blindness: $25.6 \%$ with the concept, and $52.5 \%$ with a specific example. However, knowledge of inattentional blindness was unrelated to noticing (for concept, $22.0 \%$ of noticers were familiar, $28.9 \%$ unfamiliar; $\chi^{2}=0.89, p=$ .35; for examples, $28.7 \%$ of noticers were familiar, $25.3 \%$ unfamiliar; $\chi^{2}=0.29, p=.59$ ). Consequently, we did not exclude participants on the basis of their responses to these questions. This finding has practical ramifications for future studies of inattentional blindness; provided that participants do not link the task at hand to their existing knowledge of inattentional blindness, it should be possible to test people who are already aware of the phenomenon (see Beanland \& Pammer, 2010).

As in Study 1, we conducted logistic regression to test whether tracking ability or working memory performance predicted noticing. We also tested whether tracking ability moderates the relationship between working memory and noticing via a hierarchal logistic regression analysis (entering tracking thresholds and working memory scores first, and the product of the two second). Replicating earlier evidence (Simons \& Jensen, 2009) and Study 1, tracking ability did not predict noticing of the unexpected cross $(p=$ .83). And, consistent with Study 1, working memory performance (both for the $n$-back tasks and the OSPAN task) did not predict noticing (all $p \mathrm{~s}>.15$ ). ${ }^{5}$ In fact, for the OSPAN task, the trend was opposite that of prior reports, with higher scores associated with less noticing. Finally, the interaction of tracking ability and working memory did not predict noticing (all $p \mathrm{~s}>.2$ ).

As in Study 1, tracking performance was positively associated with working memory (verbal $n$-back, $r=.17, p<.05$; spatial $n$-back, $r=.24, p<.01$; OSPAN, $r=.13, p=.07$ ), suggesting that executive control of attention is important for attentive tracking. Together, these findings suggest that the mechanisms that contribute to focused attention, working memory, and tracking do not predict the detection of unexpected objects.

\section{General discussion}

In two studies with large samples, neither tracking ability nor working memory predicted noticing of unexpected

\footnotetext{
${ }^{5}$ As in Study 1, we explored various approaches for calculating performance for the working memory tasks (e.g., using the partial scoring procedure for the OSPAN task), as well as combining scores from these three tasks (e.g., using principal components analysis), but none of these efforts yielded a significant association between working memory and noticing.
} 
objects. The lack of a relationship between attentive tracking ability and noticing of unexpected objects replicates previous work (Simons \& Jensen, 2009) and lends additional support to the claim that, while task demands do influence rates of inattentional blindness, the ability to handle those demands does not. Our finding that working memory performance is unrelated to noticing of unexpected objects is consistent with the lack of an overall association reported in one other study (Seegmiller et al., 2011). However, it is at odds with studies reporting such a relationship either in the overall analysis (Hannon \& Richards, 2010; Richards et al., 2010) or just among participants who counted accurately in the tracking task (Seegmiller et al., 2011).

Caution is merited when interpreting null findings such as ours. This is particularly true given that the same individual cannot be tested with the same unexpected object repeatedly, which precludes traditional methods for ensuring reliability. However, the discrepancy between our findings and previous reports is unlikely to result from insufficient power to find an effect. First, we replicated our finding with two independent samples and with several measures of working memory. Our measures had adequate variability to reveal a relationship - as would be expected on the basis of models of executive attention (see Engle, 2002), we found reliable correlations between working memory and tracking performance. In contrast to those associations, the differences between those who noticed the unexpected cross and those who did not were small and did not approach significance. In fact, the only trend (OSPAN scores in Study 2) was in the direction opposite the one found in previous reports. Finally, each of our samples had greater statistical power than have recent findings of a direct positive relationship. ${ }^{6}$ Although a noticing rate closer to $50 \%$ in each study would have further increased our statistical power, the fact that we obtained consistent results across two different rates of noticing $(70.7 \%$ in Study 1 vs. $27.2 \%$ in Study 2) strengthens our conclusion that working memory and noticing are unrelated.

Rather than lack of power, the discrepancy between our findings and previous reports might result from methodological differences. None of these differences alone is likely to explain all of the discrepancies, but taken together, they might. First, the studies finding an overall correlation between working memory and noticing (Hannon \& Richards, 2010; Richards et al., 2010) tested participants from a larger age range. Although

\footnotetext{
${ }^{6}$ These analyses (conducted using G*Power 3.1 software; Faul, Erdfelder, Buchner, \& Lang, 2009) revealed that we had $80 \%$ power to detect an odds ratio of .53 for Study 1 and .60 for Study 2, relative to .46 for Study 1 of Richards et al. (2010) and .49 for Hannon and Richards (2010).
}

age was not correlated with noticing in those studies, it might have interacted with working memory. In line with this speculation, Seegmiller et al. (2011) also used a college-age sample, and like us, found no overall association between working memory and noticing. Second, most previous studies linking working memory to noticing have presented the unexpected object on the first trial of the inattentional-blindness task (Hannon \& Richards, 2010; Richards et al., 2010, Study 1; Seegmiller et al., 2011). If participants with lower working memory capacity need more practice to understand the task, they might be less likely to notice the unexpected stimulus because of the challenge of comprehending the task, rather than because of greater inattentional blindness per se. In line with this idea, practice with tracking can increase noticing (Richards et al., 2010). ${ }^{7}$ Third, the relationship between working memory and noticing might depend on the salience of the unexpected stimulus. The red cross used in previous studies (Hannon \& Richards, 2010; Richards et al., 2010) was more distinctive than our gray cross. Perhaps lower working memory capacity is associated with a greater influence of physical salience (e.g., see Boot, Brockmole, \& Simons, 2005). Likewise, the positive relationship between working memory and noticing for accurate counters in the basketball task (Seegmiller et al., 2011) might be explained by the nature of the unexpected stimulus. Their participants monitored the players wearing black, so the unexpected gorilla at least partially matched their attention set for the color black. Perhaps participants with greater working memory capacity are more likely to experience contingent attention capture by features consistent with their attention set (see Most et al., 2005; Most et al., 2001; Simons \& Chabris, 1999).

Our evidence shows that the relationship between working memory and noticing, to the extent that it exists, might be limited in scope to cases in which working memory influences primary task performance or attention sets. That is, this relationship might only exist when it is moderated by other variables, and thus might not reflect a stable individual difference that applies across a range of tasks and situations. Given the practical ramifications of such a relationship (e.g., stable individual differences in noticing could be used to select security scanners or image analysts), further large-scale replications will be needed before claiming evidence for a robust individual difference in noticing.

\footnotetext{
${ }^{7}$ While that study did not find a significant interaction between practice and working memory capacity, it might have been underpowered to detect this interaction. Furthermore, the participants in the training conditions from that study received less practice with the task than did our participants.
} 
Author notes K.B. designed and conducted the experiments as part of his doctoral thesis and wrote the first draft of the manuscript. D.J.S. contributed to the theoretical discussion, the experimental design, and the writing of the manuscript.

\section{References}

Beanland, V., \& Pammer, K. (2010). Gorilla watching: Effects of exposure and expectations on inattentional blindness. In W. Christensen, E. Schier, \& J. Sutton (Eds.), ASCS09: Proceedings of the 9th conference of the australasian society for cognitive science (pp. 12-20). Sydney: Macquarie Centre for Cognitive Science. doi:10.5096/ASCS20093

Boot, W. R., Brockmole, J. R., \& Simons, D. J. (2005). Attention capture is modulated in dual-task situations. Psychonomic Bulletin \& Review, 12, 662-668. doi:10.3758/BF03196755

Chabris, C. F., Weinberger, A., Fontaine, M., \& Simons, D. J. (2011). You do not talk about Fight Club if you do not notice Fight Club: Inattentional blindness for a simulated real-world assault. $i$-Perception, 2, 150-153. doi:10.1068/10436

Conway, A. R. A., Cowan, N., \& Bunting, M. F. (2001). The cocktail party phenomenon revisited: The importance of working memory capacity. Psychonomic Bulletin \& Review, 8, 331-335. doi:10.3758/BF03196169

Conway, A. R. A., \& Kane, M. J. (2001). Capacity, control, and conflict: An individual differences perspective on attentional capture. In C. L. Folk \& B. S. Gibson (Eds.), Attraction, distraction, and action: Multiple perspectives on attentional capture (pp. 349-374). Amsterdam: Elsevier.

De Fockert, J., \& Bremner, A. J. (2011). Release of inattentional blindness by high working memory load: Elucidating the relationship between working memory and selective attention. Cognition, 121, 400-408. doi:10.1016/j.cognition.2011.08.016

Engle, R. W. (2002). Working memory capacity as executive attention. Current Directions in Psychological Science, 11, 19-23. doi:10.1111/1467-8721.00160

Faul, F., Erdfelder, E., Buchner, A., \& Lang, A.-G. (2009). Statistical power analyses using $\mathrm{G}^{*}$ Power 3.1: Tests for correlations and regression analyses. Behavior Research Methods, 41, 1149-1160. doi:10.3758/BRM.41.4.1149

Fougnie, D., \& Marois, R. (2007). Executive memory load induces inattentional blindness. Psychonomic Bulletin \& Review, 14, 142 147. doi:10.3758/BF03194041

Hannon, E. M., \& Richards, A. (2010). Is inattentional blindness related to individual differences in visual working memory capacity or executive control functioning? Perception, 39, 309-319. doi:10.1068/p6379

Kane, M. J., Conway, A. R. A., Miura, T. K., \& Colflesh, G. J. H. (2007). Working memory, attention control, and the $n$-back task: A question of construct validity. Journal of Experimental Psychology: Learning, Memory, and Cognition, 33, 615-622. doi: $10.1037 / 0278-7393.33 .3 .615$

Kane, M. J., Poole, B. J., Tuholski, S. W., \& Engle, R. W. (2006). Working memory capacity and the top-down control of visual search: Exploring the boundaries of "executive attention. Journal of Experimental Psychology: Learning, Memory, and Cognition, 32, 749-777. doi:10.1037/0278-7393.32.4.749

Lavie, N., \& De Fockert, J. (2005). The role of working memory in attention capture. Psychonomic Bulletin \& Review, 12, 669-674. doi:10.3758/BF03196756

Mack, A., \& Rock, I. (1998). Inattentional blindness. Cambridge: MIT Press.

Most, S. B., Scholl, B. J., Clifford, E. R., \& Simons, D. J. (2005). What you see is what you set: Sustained inattentional blindness and the capture of awareness. Psychological Review, 112, 217-242. doi:10.1037/0033-295X.112.1.217

Most, S. B., \& Simons, D. J. (2001). Attention capture, orienting, and awareness. In C. L. Folk \& B. S. Gibson (Eds.), Attraction, distraction, and action: Multiple perspectives on attentional capture (pp. 151-176). Amsterdam: Elsevier.

Most, S. B., Simons, D. J., Scholl, B. J., Jimenez, R., Clifford, E., \& Chabris, C. F. (2001). How not to be seen: The contribution of similarity and selective ignoring to sustained inattentional blindness. Psychological Science, 12, 9-17. doi:10.1111/1467-9280.00303

Owen, A. M., McMillan, K. M., Laird, A. R., \& Bullmore, E. (2005). $\mathrm{N}$-back working memory paradigm: A meta-analysis of normative functional neuroimaging studies. Human Brain Mapping, 25, 4659. doi:10.1002/hbm.20131

Richards, A., Hannon, E., \& Derakshan, N. (2010). Predicting and manipulating the incidence of inattentional blindness. Psychological Research, 74, 513-523. doi:10.1007/s00426-0090273-8

Seegmiller, J. K., Watson, J. M., \& Strayer, D. L. (2011). Individual differences in susceptibility to inattentional blindness. Journal of Experimental Psychology: Learning, Memory, and Cognition, 37, 785-791. doi:10.1037/a0022474

Simons, D. J., \& Chabris, C. F. (1999). Gorillas in our midst: Sustained inattentional blindness for dynamic events. Perception, 28, 1059 1074. doi:10.1068/p2952

Simons, D. J., \& Jensen, M. S. (2009). The effects of individual differences and task difficulty on inattentional blindness. Psychonomic Bulletin \& Review, 16, 398-403. doi:10.3758/ PBR.16.2.398

Strayer, D. L., \& Drews, F. A. (2007). Cell-phone-induced driver distraction. Current Directions in Psychological Science, 16, 128-131. doi:10.1111/j.1467-8721.2007.00489.x

Todd, J. J., Fougnie, D., \& Marois, R. (2005). Visual short-term memory load suppresses tempo-parietal junction activity and induces inattentional blindness. Psychological Science, 16, 965972. doi:10.1111/j.1467-9280.2005.01645.x

Unsworth, N., \& Engle, R. W. (2007). The nature of individual differences in working memory capacity: Active maintenance in primary memory and controlled search from secondary memory. Psychological Review, 114, 104-132. doi:10.1037/ 0033-295X.114.1.104

Unsworth, N., Heitz, R. P., Schrock, J. C., \& Engle, R. W. (2005). An automated version of the operation span task. Behavior Research Methods, 37, 498-505. doi:10.3758/ BF03192720 